\title{
Specific Diversity and Dynamics of the Phytoplankton Population in a Pond Fertilized with Chicken Droppings in the West Highlands Cameroon
}

\author{
Algrient $\mathrm{NT}^{1 *}$, Berlin $\mathrm{S}^{1}$, Thomas $\mathrm{EE}^{1}$, Aimerance $\mathrm{KD}^{1}$, Francis $\mathrm{KM}^{2}$ and \\ Tchoumboue ${ }^{1}$ \\ ${ }^{1}$ Ichtiology and Applied Hydrobology Research Unit, Faculty of Agronomy and Agricultural \\ Sciences, University of Dschang, Cameroon \\ ${ }^{2}$ Applied Botany Research Units, Faculty of Sciences, University of Dschang, Cameroon
}

\section{Research Article \\ Volume 4 Issue 3}

Received Date: June 21, 2020

Published Date: July 03, 2020

DOI: $10.23880 /$ ijoac-16000192

*Corresponding author: Nana Towa Algrient, Ichtiology and Applied Hydrobology

Research Unit, Department of Forestry, Faculty of Agronomy and Agricultural Sciences, University of Dschang, Cameroon, Tel: +237673180089; Email: algrient@yahoo.fr

\section{Abstract}

Phytoplankton is one of the essential compartments of the food chain in aquatic ecosystems, especially in fish farming ponds. In order to contribute to the improvement of fish productivity in ponds by the production of phytoplankton through fertilization, a study was carried out at the Research and Application Farm, more precisely at the Aquaculture Station of the University of Dschang. The study was conducted in 9 derivation ponds of the same surface area $(5.7 \times 5.7 \mathrm{~m})$ and $1 \mathrm{~m}$ depth with a flow rate of 3.23 litre/minute. Mesh net $(1.5 \mathrm{~mm})$ was attached to the inlet pipe of each pond to prevent fish intrusion from the reservoir. Each of the three treatments consisting of 0; 800 and $1000 \mathrm{~kg} / \mathrm{ha}$ of chicken droppings was applied to one of the ponds randomly. Each treatment was repeated three times. Phytoplankton and water quality data were collected every two weeks. The results showed a richness of 237 species grouped in 6 phytoplankton phylum that were registered independently of the dropping dose. This species richness decreased with increase of the dropping dose either, 164; 121 and 109 species corresponding to doses $0 ; 800$ and $1000 \mathrm{~kg} /$ ha respectively. On the other hand, density values were lowest in unfertilized ponds and highest in ponds fertilized with the highest dose $(1000 \mathrm{~kg} / \mathrm{ha})$. For the production of phytoplanktonophagous fish the dose of $1000 \mathrm{~kg}$ could be used. However, it would be important to search for the optimal weekly fertilization dose.

Keywords: Phytoplankton; Diversity; Density; Chicken Droppings

\section{Introduction}

Phytoplankton is made up of all the plant microorganisms suspended in the water, capable to product their own organic substance by photosynthesis, using solar energy, water, carbonic acid gas and nutrient salts. It is one of the essential compartments that condition the food chain in aquatic ecosystems, especially in fish farming ponds. Thus, in aquaculture production, the growth of fish, especially common carp and tilapia, depends on the autotrophic pathway and so on phytoplankton for between 50 and $80 \%$ of their growth. The organisms that make up phytoplankton are ecologically tolerant. Their development and stability depend essentially on the nutrient richness of the environment, especially phosphorus and nitrogen, and on the grazing of phytoplanktonophagous [1].

In China, waste from all sources has been spread in fish ponds for two millennia. It is the same with dropping, which is traditionally brought into fish nursery ponds to 
stimulate phytoplankton development. These practices have led several scientists to conduct studies on phytoplankton production in fish ponds. Barbe, et al. [2] have studied usage of phytoplankton to estimate the potential fish production in fish ponds. Other work has focused on monitoring phytoplankton in lakes, rivers and uncontrolled environments [3-7]. However, few works has been done on the management of phytoplankton organisms in earthen fish ponds despite the fact that they represent the most widely used infrastructure $(70 \%)$ in the world. Moreover, none of this work has focused on the effect of the amount of fertilizer regularly applied in ponds on phytoplankton development.

The objective of this work is to contribute to the improvement of fish productivity by the production of phytoplankton through pond fertilization. More specifically, the aim is to evaluate the effect of the dose of chicken droppings on the richness, density and dynamics of phytoplankton development.

\section{Materials and Methods}

\section{Area and Period of Study}

The test was carried out at the Aquaculture Station of the Application and Research Farm (F.A.R) of the University of Dschang (North Latitude: $5^{\circ} 44^{\prime}-5^{\circ} 36^{\prime}$ and Est Longitude: $10^{\circ} 06^{\prime}-9^{\circ} 85^{\prime}$, altitude: $1392-1396 \mathrm{~m}$ ) located in the West Cameroon highlands agro-ecological zone. This zone is characterized by a short dry season (mid-November to mid-March) and a long rainy season (mid-March to midNovember). Annual rainfall varies between 1500 and 2000 $\mathrm{mm}$ and temperatures range between $14^{\circ} \mathrm{C}$ (July-August) and $25^{\circ} \mathrm{C}$ (February).

\section{Obtaining and Conditioning of Chicken Droppings}

In order to avoid variability in chemical composition, hen droppings were collected from the same farm and stored at room temperature. A sample was taken to determine the concentration of dry matter, total nitrogen and total phosphorus. The mean values of the dropping characteristics were as follows: dry matter $(80.2 \pm 3.33 \%)$, total nitrogen $(2$ $\pm 0.14 \%$ ) and total phosphorus (1.5 $\pm 0.06 \%)$.

\section{Experimental Ponds}

The test was carried out inside 9 diversion ponds with the same surface area $(5.7 \times 5.7 \mathrm{~m})$ and a depth of $1 \mathrm{~m}$ with a flow rate of 3.23 liters per minute. In order to eliminate undesirable organisms (fish) and increase the alkalinity of the environment, the ponds were dewatered for a period of 7 days and limed with quicklime at a dose of $400 \mathrm{~kg} / \mathrm{ha}$. Mesh size net $(1.5 \mathrm{~mm})$ was attached to the inlet pipe of each pond to prevent fish intrusion from the reservoir. Each of the three treatments consisting of $0 ; 800$ and $1000 \mathrm{~kg} / \mathrm{ha}$ of chicken droppings was applied in one of the ponds randomly. Each treatment was repeated three times.

\section{Conduct of the Trial and Data Collection}

Pond Fertilization: In order to control phytoplankton production, chicken droppings were applied to the ponds by spreading once a week for a period of 6 months. For this purpose, the fertilizer doses were weighed with an electronic precision scale $0.1 \mathrm{~g}$ each time before application.

Sampling of Phytoplankton Organisms: Phytoplankton sampling was carried out on a biweekly basis for 6 months. Sampling was carried out at 20 different points in the water column of each pond using a calibrated polyethylene container with a capacity of 1 liter, i.e. a total volume of 20 liters/pond filtered through a $40 \mu \mathrm{m}$ mesh plankton sieve. A volume of $350 \mathrm{ml}$ of concentrated phytoplankton was recovered, fixed by the addition of $5 \%$ formaldehyde and kept in plastic bottles for quantitative and qualitative analyses.

Determination of the Physico-Chemical Characteristics of Water: The Secchi disc transparency, temperature, $\mathrm{pH}$, dissolved oxygen and electrical conductivity were measured directly in the field using a ballasted Secchi disc attached to a graduated string, Thermo-Conductimeter, Thermo$\mathrm{PH}$ meter, $\mathrm{pH}$ meter, Thermo-Oxymeter and ThermoConductimeter from HANNA respectively. Nitrite, nitrate and total phosphates were determined by spectrophotometry (spectrophotometer HACH DR/2000) according to Apha techniques [8].

Qualitative and Quantitative Analysis of Phytoplankton: Each previously filtered sample $(350 \mathrm{ml})$ was left to rest for a minimum of 24 hours to allow for phytoplankton sedimentation. A volume of $300 \mathrm{ml}$ of water was then removed above the phytoplankton residue by siphoning and the concentrated phytoplankton samples were kept in beakers. The qualitative analysis of the phytoplankton was carried out according to the method described by Nguetsop, et al. [9]. Thus, after homogenization, two drops (10 $\mu \mathrm{l})$ of each concentrated phytoplankton sample were taken with a pipette, mounted between slide and slide and observed using an Olympus optical microscope ( $\times 40$ objectives), model BH2 , equipped with a nomarski lens. For each sample, three slides were prepared to ensure reproducibility of the slides [9].

The identifications were made using the keys of Bourelly and Manguin [10]; Compère [11]; Couté and Rousselin [12], Compère [13,14]; Gasse [15]; Iltis [16]; Gasse [17]; Krammer and Lange-Bertalot [18].

The cell counts were performed using an inverted 
microscope, ZEISS 471202 with the $\times 40$ objective as recommended by Lund, et al. [19]. After homogenization, 10 $\mathrm{ml}$ of the sample was taken with a calibrated pipette, placed in a settling cup and allowed to settle for $10 \mathrm{~min}$. Counts were made on six fields taken randomly from the cup. The minimum number of units (cell, filament, cenobe or colony) counted per replicate of a sample was set at 400 , in order to have an accuracy of \pm 10 to $95 \%$ confidence interval.

\section{Statistical Analyses}

The collected data were subjected to the one-factor analysis of variance (ANOVA 1). In the case of significant differences between the means, the Duncan's test was applied to separate them at the 5\% significance level. SPSS 20.0 (Statistical Package for Social Sciences) was used for these analyses.

\section{Results}

\section{Effects of Chicken Droppings Dose on Phytoplankton Species Richness and Distribution}

The composition of the phytoplankton stand summarized in Table 1 shows that, independently of the doses of chicken droppings, a total of 237 species grouped in 6 phytoplankton phylum were recorded.

The distribution of phytoplankton species shows that of the 237 species identified, 164 are represented in unfertilized ponds, i.e. a proportion of $61.19 \%$. As regards fertilized ponds, a total of 121 species or $51.05 \%$ were represented in ponds having received $800 \mathrm{~kg}$ of dropping and 109 species representing $46 \%$ in $1000 \mathrm{~kg}$.

From Table 1 summarizing the distribution of species, it can be seen that 83 out of 237 species were represented only in unfertilized ponds. The dominant species in these ponds are Merismopedia elegans, Mycrocystis aeruginosa, Nostoc entophytum.

In the fertilized ponds, the species specifically represented in the $800 \mathrm{~kg}$ treatment were the highest $(23$ species out of 237) compared to those recorded with the $1000 \mathrm{~kg}$ of chicken droppings dose (23 species). It should be noted that 66 species (i.e. $27.84 \%$ of the total species recorded) were represented transversally in all three treatments $(0 ; 800$ and $1000 \mathrm{~kg})$.

The Pyrrophyte phylum is only represented in the ponds fertilized with the highest dose of chicken dropping, whereas the Rhodophyte phylum is only represented in the treatment with $800 \mathrm{~kg} / \mathrm{ha}$ of chicken dropping.

\begin{tabular}{|c|c|c|c|}
\hline \multirow{2}{*}{ Phytoplankton taxa } & \multicolumn{3}{|c|}{ Phytoplankton taxa (kg) } \\
\hline & $\mathbf{0}$ & 800 & 1000 \\
\hline \multicolumn{4}{|c|}{ Cyanophytes } \\
\hline Pseudanabaena catenata & $\mathrm{X}$ & $\mathrm{X}$ & $\mathrm{X}$ \\
\hline Merismopedia elegans & $\mathrm{X}$ & - & - \\
\hline Tolypothrix distorta & $\mathrm{X}$ & $\mathrm{X}$ & $\mathrm{X}$ \\
\hline Calothrix scytonemicola & - & $\mathrm{X}$ & - \\
\hline Calothrix columbiana & - & - & $\mathrm{X}$ \\
\hline Microcystis robusta & - & $\mathrm{X}$ & - \\
\hline Microcystis aeruginosa & $\mathrm{X}$ & - & - \\
\hline Nostoc entophytum & $\mathrm{X}$ & - & - \\
\hline Lyngbya bargentii & $\mathrm{X}$ & - & - \\
\hline Oscillatoria chlorina & $\mathrm{X}$ & - & - \\
\hline Oscillatoria bornetii & - & - & $\mathrm{X}$ \\
\hline Gomphosphaeria pusilla & $\mathrm{X}$ & - & - \\
\hline \multicolumn{4}{|c|}{ Chlorophytes } \\
\hline Pediastrum duplex & $\mathrm{X}$ & $\mathrm{X}$ & $\mathrm{X}$ \\
\hline Trentepohlia bossei & - & $\mathrm{X}$ & - \\
\hline
\end{tabular}




\begin{tabular}{|c|c|c|c|}
\hline Chodatella quadriseta & $\mathrm{X}$ & - & - \\
\hline Eremosphera gigas & - & $\mathrm{X}$ & - \\
\hline Micratinium pusillum & - & $\mathrm{X}$ & - \\
\hline Oedogonium nasatum & - & $\mathrm{X}$ & - \\
\hline Carteria multifilis & $\mathrm{X}$ & - & - \\
\hline Scenedesmus armatus & $\mathrm{X}$ & - & - \\
\hline Scenedesmus opaliensis & $\mathrm{X}$ & - & - \\
\hline Scenedesmus quadricauda var longispina & - & $\mathrm{X}$ & - \\
\hline Scenedesmus quadricauda & - & $\mathrm{X}$ & - \\
\hline Scenedesmus brasiliensis & $\mathrm{X}$ & - & - \\
\hline Senedesmus armatus var bicaudatus & $\mathrm{X}$ & - & - \\
\hline Scenedesmus acutiformis & $\mathrm{X}$ & - & - \\
\hline Scenedesmus obtunus f. ecornis & - & $\mathrm{X}$ & - \\
\hline Ulothrix zonata & $\mathrm{X}$ & - & - \\
\hline Ulothrix subtllissima & $\mathrm{X}$ & - & - \\
\hline Netriumdigitus var naegel & - & - & $\mathrm{X}$ \\
\hline Spirotaenia condensata & $\mathrm{X}$ & $\mathrm{X}$ & $\mathrm{X}$ \\
\hline Desmidium aequale & $\mathrm{X}$ & - & - \\
\hline Pleurotaenium nodosum & $\mathrm{X}$ & - & - \\
\hline Pleurotaenium sp. & - & $\mathrm{X}$ & $\mathrm{X}$ \\
\hline Pleurotaenium cylindricum var stuhlmannii & $\mathrm{X}$ & - & - \\
\hline Pleurotaenium elatum var camerounense & - & - & $\mathrm{X}$ \\
\hline Pleurotaenium clavatum & $\mathrm{X}$ & $\mathrm{X}$ & $\mathrm{X}$ \\
\hline Pleurotaenium clavatum var elongatum & $\mathrm{X}$ & $\mathrm{X}$ & $\mathrm{X}$ \\
\hline Mougeotia sp. & $\mathrm{X}$ & $\mathrm{X}$ & $\mathrm{X}$ \\
\hline Mougeotia drouetii & $\mathrm{X}$ & $\mathrm{X}$ & $\mathrm{X}$ \\
\hline Closterium annae & $\mathrm{X}$ & - & - \\
\hline Closterium calosporum var majus & - & - & $\mathrm{X}$ \\
\hline Closterium cornu var javanicum & - & - & $\mathrm{X}$ \\
\hline Closterium dianae & - & - & $\mathrm{X}$ \\
\hline Closterium dianae var brevius & $\mathrm{X}$ & - & - \\
\hline Closterium ehrenbergii & - & $\mathrm{X}$ & - \\
\hline Closterium exile & $\mathrm{X}$ & $\mathrm{X}$ & $\mathrm{X}$ \\
\hline Closterium kuetzingii & - & $\mathrm{X}$ & $\mathrm{X}$ \\
\hline Closterium moniliferum & $\mathrm{X}$ & $\mathrm{X}$ & $\mathrm{X}$ \\
\hline Closterium nasatum & $\mathrm{X}$ & $\mathrm{X}$ & $\mathrm{X}$ \\
\hline Closterium parvulum & - & $\mathrm{X}$ & - \\
\hline Closterium parvulum f. majus & $\mathrm{X}$ & $\mathrm{X}$ & - \\
\hline
\end{tabular}




\begin{tabular}{|c|c|c|c|}
\hline Closterium ralfsii var hybridum & $\mathrm{X}$ & - & - \\
\hline Closterium $s p$ & $\mathrm{X}$ & - & - \\
\hline Closterium tumidium var myladicum & - & $\mathrm{X}$ & - \\
\hline Closterium tumidum & $\mathrm{X}$ & $\mathrm{X}$ & $\mathrm{X}$ \\
\hline Cosmarium angulonum & - & $\mathrm{X}$ & - \\
\hline Cosmarium connatum & $\mathrm{X}$ & - & - \\
\hline Cosmarium gerdae & - & $\mathrm{X}$ & - \\
\hline Cosmarium granatum & - & - & $\mathrm{X}$ \\
\hline Cosmarium laeve & $\mathrm{X}$ & - & - \\
\hline Cosmarium magnificum & $\mathrm{X}$ & - & - \\
\hline Cosmarium margaritatum & $\mathrm{X}$ & - & - \\
\hline Cosmarium monodii & $\mathrm{X}$ & - & - \\
\hline Cosmarium monomazum & $\mathrm{X}$ & - & - \\
\hline Cosmarium parolalis & $\mathrm{X}$ & - & - \\
\hline Cosmarium quadrum & - & $\mathrm{X}$ & - \\
\hline Cosmarium scottii & $\mathrm{X}$ & - & - \\
\hline Cosmarium sp. & $\mathrm{X}$ & - & - \\
\hline Cosmarium subamiculatum & $\mathrm{X}$ & - & - \\
\hline Cosmarium vogesiacum & $\mathrm{X}$ & - & - \\
\hline Cosmarium vogesiacum var f. bipunctatum & $\mathrm{X}$ & - & - \\
\hline Euastrum pectinatum & $\mathrm{X}$ & $\mathrm{X}$ & $\mathrm{X}$ \\
\hline Euastrum spinolosum, forma & $\mathrm{X}$ & $\mathrm{X}$ & $\mathrm{X}$ \\
\hline Euastrum sphyroides var heironymusii & $\mathrm{X}$ & $\mathrm{X}$ & $\mathrm{X}$ \\
\hline Euastrumspinolosum var lindae & $\mathrm{X}$ & - & - \\
\hline Euastrumdivergens var bourrellyanum & $\mathrm{X}$ & $\mathrm{X}$ & $\mathrm{X}$ \\
\hline Staurastrum cyclacanthum var. ubacathum & $\mathrm{X}$ & $\mathrm{X}$ & - \\
\hline Staurastrum inflexum, forma & $\mathrm{X}$ & $\mathrm{X}$ & $\mathrm{X}$ \\
\hline Staurastrum asterias & $\mathrm{X}$ & $\mathrm{X}$ & $\mathrm{X}$ \\
\hline Staurastrum zonatum var productum & $\mathrm{X}$ & - & - \\
\hline Staurastrum pingue & $\mathrm{X}$ & - & - \\
\hline Staurastrum tetracerum var subexcavatum & $\mathrm{X}$ & $\mathrm{X}$ & $\mathrm{X}$ \\
\hline Staurastrum polymorphum & $\mathrm{X}$ & $\mathrm{X}$ & $\mathrm{X}$ \\
\hline Micrasterias truncate & $\mathrm{X}$ & - & - \\
\hline Micrasterias sp. & - & $\mathrm{X}$ & $\mathrm{X}$ \\
\hline Micraterias radiosa f. minuta & $\mathrm{X}$ & $\mathrm{X}$ & $\mathrm{X}$ \\
\hline Micrasterias foliacae & $\mathrm{X}$ & $\mathrm{X}$ & $\mathrm{X}$ \\
\hline Micrasterias mahabules harenis var comperei & $\mathrm{X}$ & - & - \\
\hline Micrasterias crux-melitensis & $\mathrm{X}$ & $\mathrm{X}$ & $\mathrm{X}$ \\
\hline
\end{tabular}




\begin{tabular}{|c|c|c|c|}
\hline Micrasterias radians & $\mathrm{X}$ & $\mathrm{X}$ & $\mathrm{X}$ \\
\hline Spirogyra puncticulata, forma & - & $\mathrm{X}$ & - \\
\hline Spirogyra parangabae & - & $\mathrm{X}$ & - \\
\hline Spirogyra liana & - & $\mathrm{X}$ & - \\
\hline Spirogyra verrucasa & - & $\mathrm{X}$ & - \\
\hline Spirogyra neglecta & $\mathrm{X}$ & $\mathrm{X}$ & $\mathrm{X}$ \\
\hline Spirogyra hollandiae & - & $\mathrm{X}$ & $\mathrm{X}$ \\
\hline Spirogyra decimina & - & $\mathrm{X}$ & $\mathrm{X}$ \\
\hline Spirogyra setiformis & $\mathrm{X}$ & $\mathrm{X}$ & $\mathrm{X}$ \\
\hline Spirogyra maxima & - & $\mathrm{X}$ & - \\
\hline Spirogyra irregularis & $\mathrm{X}$ & $\mathrm{X}$ & $\mathrm{X}$ \\
\hline Spirogyra crassa & - & - & - \\
\hline Spirogyra fluviatilis & - & - & $\mathrm{X}$ \\
\hline Spirogyra majuscula & - & $\mathrm{X}$ & - \\
\hline Spirogyra weberi & - & $\mathrm{X}$ & $\mathrm{X}$ \\
\hline Spirogyr apseudoneglecta & $\mathrm{X}$ & $\mathrm{X}$ & $\mathrm{X}$ \\
\hline Spirogyra variformis & $\mathrm{X}$ & $\mathrm{X}$ & $\mathrm{X}$ \\
\hline Spirogyra asp. & $\mathrm{X}$ & $\mathrm{X}$ & $\mathrm{X}$ \\
\hline Spirogyra gracilis & $\mathrm{X}$ & $\mathrm{X}$ & $\mathrm{X}$ \\
\hline Spirogyra varians & $\mathrm{X}$ & - & - \\
\hline Spirogyra corrugata & $\mathrm{X}$ & $\mathrm{X}$ & $\mathrm{X}$ \\
\hline Spirogyra reflexa & - & - & $\mathrm{X}$ \\
\hline Spirogyra chakiensis f. major & $\mathrm{X}$ & - & - \\
\hline Gonatozygon $s p$ & $\mathrm{X}$ & $\mathrm{X}$ & $\mathrm{X}$ \\
\hline Gonatozygon brebisonii & - & $\mathrm{X}$ & - \\
\hline Gonatozygon monotaenium var pilossellum & $\mathrm{X}$ & - & - \\
\hline Gonatozygon kinahanii & $\mathrm{X}$ & $\mathrm{X}$ & $\mathrm{X}$ \\
\hline Gonatozygon monotaenium & $\mathrm{X}$ & $\mathrm{X}$ & $\mathrm{X}$ \\
\hline Gonatozygon aculeatum & $\mathrm{X}$ & $\mathrm{X}$ & $\mathrm{X}$ \\
\hline Zygnema sp. & $\mathrm{X}$ & $\mathrm{X}$ & $\mathrm{X}$ \\
\hline \multicolumn{4}{|c|}{ Bacillariophytes } \\
\hline Amphora libyca & $\mathrm{X}$ & - & - \\
\hline Amphora ovalis & $\mathrm{X}$ & $\mathrm{X}$ & $\mathrm{X}$ \\
\hline Amphora inariensis & $\mathrm{X}$ & - & - \\
\hline Amphora sp. & $\mathrm{X}$ & - & - \\
\hline Diploneis sp. & $\mathrm{X}$ & - & - \\
\hline Stauroneis undata & $\mathrm{X}$ & - & - \\
\hline Cymbella caespitora & $\mathrm{X}$ & - & - \\
\hline
\end{tabular}




\begin{tabular}{|c|c|c|c|}
\hline Cymbella gaemanii & $\mathrm{X}$ & $\mathrm{X}$ & $\mathrm{X}$ \\
\hline Cymbella hustedtii & $\mathrm{X}$ & $\mathrm{X}$ & $\mathrm{X}$ \\
\hline Cymbella minuta & - & $\mathrm{X}$ & - \\
\hline Cymbella naviculiformis & $\mathrm{X}$ & $\mathrm{X}$ & $\mathrm{X}$ \\
\hline Cymbella prostrata & - & - & $\mathrm{X}$ \\
\hline Cymbellasilesiaca & $\mathrm{X}$ & - & - \\
\hline Cymbella sp & $\mathrm{X}$ & $\mathrm{X}$ & $\mathrm{X}$ \\
\hline Gomphonema africanum & $\mathrm{X}$ & $\mathrm{X}$ & $\mathrm{X}$ \\
\hline Gomphonema amoenum & $\mathrm{X}$ & - & - \\
\hline Gomphonema angustum & $\mathrm{X}$ & $\mathrm{X}$ & $\mathrm{X}$ \\
\hline Gomphonema clavatum & $\mathrm{X}$ & $\mathrm{X}$ & $\mathrm{X}$ \\
\hline Gomphonema elongatum & - & $\mathrm{X}$ & - \\
\hline Gomphonema parvulum & $\mathrm{X}$ & $\mathrm{X}$ & $\mathrm{X}$ \\
\hline Gomphonema puiggarianum & $\mathrm{X}$ & - & - \\
\hline Gomphonema minutum & $\mathrm{X}$ & - & - \\
\hline Navicula elginensis & - & $\mathrm{X}$ & - \\
\hline Navicula halophila & - & - & $\mathrm{X}$ \\
\hline Navicula mutica $f$. intermedia & - & - & - \\
\hline Navicula amphibola & $\mathrm{X}$ & $\mathrm{X}$ & $\mathrm{X}$ \\
\hline Navicula cryptocephala & $\mathrm{X}$ & - & - \\
\hline Navicula pseudogrimmei & $\mathrm{X}$ & - & - \\
\hline Navicula americana & $\mathrm{X}$ & $\mathrm{X}$ & $\mathrm{X}$ \\
\hline Navicula evanida & $\mathrm{X}$ & - & $\mathrm{X}$ \\
\hline Naviculla sp & $\mathrm{X}$ & $\mathrm{X}$ & $\mathrm{X}$ \\
\hline Navicula radiosa & - & $\mathrm{X}$ & - \\
\hline Navicula consentanea & $\mathrm{X}$ & $\mathrm{X}$ & $\mathrm{X}$ \\
\hline Navicula pusila & $\mathrm{X}$ & $\mathrm{X}$ & $\mathrm{X}$ \\
\hline Navicula perminuta & $\mathrm{X}$ & - & - \\
\hline Navicula explanata & - & $\mathrm{X}$ & $\mathrm{X}$ \\
\hline Pinnularia acrosphaeria & $\mathrm{X}$ & $\mathrm{X}$ & $\mathrm{X}$ \\
\hline Pinnularia undulata & $\mathrm{X}$ & - & - \\
\hline Pinnularia ignobilis & $\mathrm{X}$ & $\mathrm{X}$ & $\mathrm{X}$ \\
\hline Pinnularia gibba var mesogonyla & $\mathrm{X}$ & - & - \\
\hline Pinnularia gibba & $\mathrm{X}$ & $\mathrm{X}$ & $\mathrm{X}$ \\
\hline Pinnularia legumen & $\mathrm{X}$ & $\mathrm{X}$ & $\mathrm{X}$ \\
\hline Pinnularia macilenta & $\mathrm{X}$ & - & - \\
\hline Frustulia rhomboides & $\mathrm{X}$ & $\mathrm{X}$ & $\mathrm{X}$ \\
\hline Fragilaria brevistriata & $\mathrm{X}$ & - & - \\
\hline
\end{tabular}




\begin{tabular}{|c|c|c|c|}
\hline Fragilaria leptostauron var dubia & - & - & $\mathrm{X}$ \\
\hline Fragilaria capucina & $\mathrm{X}$ & $\mathrm{X}$ & $\mathrm{X}$ \\
\hline Fragilaria $s p$ & $\mathrm{X}$ & $\mathrm{X}$ & $\mathrm{X}$ \\
\hline Tabelaria sp & - & - & $\mathrm{X}$ \\
\hline Meridion circulare & - & - & $\mathrm{X}$ \\
\hline Rhopalodia acuminata & $\mathrm{X}$ & - & - \\
\hline Rhopalodia rupestris & $\mathrm{X}$ & - & - \\
\hline Rhoppalodia gibberula & $\mathrm{X}$ & - & - \\
\hline Rhoppalodia bubissonii & $\mathrm{X}$ & - & - \\
\hline Rhopalodia brebissonii & $\mathrm{X}$ & - & - \\
\hline Eunotia siolii & $\mathrm{X}$ & - & $\mathrm{X}$ \\
\hline Eunotia $s p$ & $\mathrm{X}$ & $\mathrm{X}$ & - \\
\hline Surirella suecica & $\mathrm{X}$ & - & - \\
\hline Surirella splandida & $\mathrm{X}$ & - & - \\
\hline Surirella tchadensis & $\mathrm{X}$ & - & - \\
\hline Surirella robusta & $\mathrm{X}$ & - & - \\
\hline Surirella $s p$ & $\mathrm{X}$ & - & - \\
\hline Surirella elegans & $\mathrm{X}$ & $\mathrm{X}$ & $\mathrm{X}$ \\
\hline Surirella capronii & $\mathrm{X}$ & - & - \\
\hline Surirella ovalis & $\mathrm{X}$ & - & - \\
\hline Surirella brebissonii & - & $\mathrm{X}$ & - \\
\hline Melosira arenaria & $\mathrm{X}$ & $\mathrm{X}$ & $\mathrm{X}$ \\
\hline Melosira granulata & $\mathrm{X}$ & - & - \\
\hline Melosira undulata & $\mathrm{X}$ & - & - \\
\hline Aulacoseira tethera & - & $\mathrm{X}$ & - \\
\hline Aulacoseira subartica & - & - & $\mathrm{X}$ \\
\hline Aulacauseira canadensis & - & $\mathrm{X}$ & - \\
\hline Aulacoseira crenulata & $\mathrm{X}$ & - & - \\
\hline Aulacoseira distans & $\mathrm{X}$ & $\mathrm{X}$ & $\mathrm{X}$ \\
\hline Hontzschia amphioxys & - & $\mathrm{X}$ & - \\
\hline \multicolumn{4}{|c|}{ Euglenophytes } \\
\hline Euglena variabilis & $\mathrm{X}$ & $\mathrm{X}$ & $\mathrm{X}$ \\
\hline Euglena oxyuris & $\mathrm{X}$ & - & - \\
\hline Euglena limnopohila & $\mathrm{X}$ & - & - \\
\hline Euglena texta & $\mathrm{X}$ & - & - \\
\hline Euglena sp & - & - & $\mathrm{X}$ \\
\hline Euglena oxyuris f. minima & - & $\mathrm{X}$ & $\mathrm{X}$ \\
\hline Euglena tripteris var. klebsii & $\mathrm{X}$ & - & - \\
\hline
\end{tabular}




\begin{tabular}{|c|c|c|c|}
\hline Euglena oxyuris f. Playfairii & $\mathrm{X}$ & $\mathrm{X}$ & $\mathrm{X}$ \\
\hline Euglena geniculata & - & $\mathrm{X}$ & - \\
\hline Euglena ehrenbergii & $\mathrm{X}$ & - & - \\
\hline Euglena spirogyra & $\mathrm{X}$ & - & - \\
\hline Lepocinclis acuminata & $\mathrm{X}$ & $\mathrm{X}$ & $\mathrm{X}$ \\
\hline Lepocinclis fusiformis f. lemmermannii & - & $\mathrm{X}$ & - \\
\hline Lepocinclis ovum & - & $\mathrm{X}$ & $\mathrm{X}$ \\
\hline Lepocinclis salina & $\mathrm{X}$ & - & - \\
\hline Lepocinclis sp & - & - & $\mathrm{X}$ \\
\hline Phacus platalae & $\mathrm{X}$ & $\mathrm{X}$ & $\mathrm{X}$ \\
\hline Phacus applanatus & $\mathrm{X}$ & - & - \\
\hline Phacus quadricauda & $\mathrm{X}$ & - & - \\
\hline Phacus pleuronecte & $\mathrm{X}$ & - & - \\
\hline Phagus tortus & $\mathrm{X}$ & $\mathrm{X}$ & $\mathrm{X}$ \\
\hline Phacus sp & - & $\mathrm{X}$ & $\mathrm{X}$ \\
\hline Phacus lemmermannii & - & $\mathrm{X}$ & $\mathrm{X}$ \\
\hline Phacus orbicularis & $\mathrm{X}$ & $\mathrm{X}$ & $\mathrm{X}$ \\
\hline Phacus longicauda & $\mathrm{X}$ & $\mathrm{X}$ & $\mathrm{X}$ \\
\hline Trachelomonas african avar. pulchella & - & $\mathrm{X}$ & - \\
\hline Trachelomonas conica & $\mathrm{X}$ & $\mathrm{X}$ & $\mathrm{X}$ \\
\hline Trachelomonas hispida f. Minor & - & - & $\mathrm{X}$ \\
\hline Trachelomonas hispida var duplex & - & - & $\mathrm{X}$ \\
\hline Trachelomonas planctonica & $\mathrm{X}$ & - & - \\
\hline Trachelomonas robusta & $\mathrm{X}$ & $\mathrm{X}$ & $\mathrm{X}$ \\
\hline Trachelomonas verrucosa & $\mathrm{X}$ & $\mathrm{X}$ & $\mathrm{X}$ \\
\hline Trachelomonas verrucosa var granulosa & - & $\mathrm{X}$ & - \\
\hline Trachelomonas volvocina var punctata & $\mathrm{X}$ & - & - \\
\hline Astasia velox & - & $\mathrm{X}$ & $\mathrm{X}$ \\
\hline Astasia acus & $\mathrm{X}$ & - & - \\
\hline Distigma proteus & $\mathrm{X}$ & - & - \\
\hline \multicolumn{4}{|c|}{ Pyrrophytes } \\
\hline Peridinium $s p$ & - & - & $\mathrm{X}$ \\
\hline Peridinium cinctum & - & - & $\mathrm{X}$ \\
\hline Peridinium volzii & - & - & $\mathrm{X}$ \\
\hline Sphaerodinium polonicum & - & - & $\mathrm{X}$ \\
\hline \multicolumn{4}{|c|}{ Rhodophytes } \\
\hline Thoreara mosissima & - & $\mathrm{X}$ & - \\
\hline
\end{tabular}

Table 1: Distribution of phytoplankton taxa according to the droppings dose The cross ( $\mathrm{x}$ ) in each box indicates the presence of the species. 


\section{Phylum Distribution of Phytoplankton Species as a Function of Dropping Dose}

Figure 1 illustrates the distribution of species by phylum as a function of dropping dose. Overall, in unfertilized ponds, the algal flora is dominated by species belonging to the Bacillariophytes phylum (34\%) whereas ponds fertilized at 800 and $1000 \mathrm{~kg}$ were dominated by Charophytes at 42 and $30 \%$ respectively.

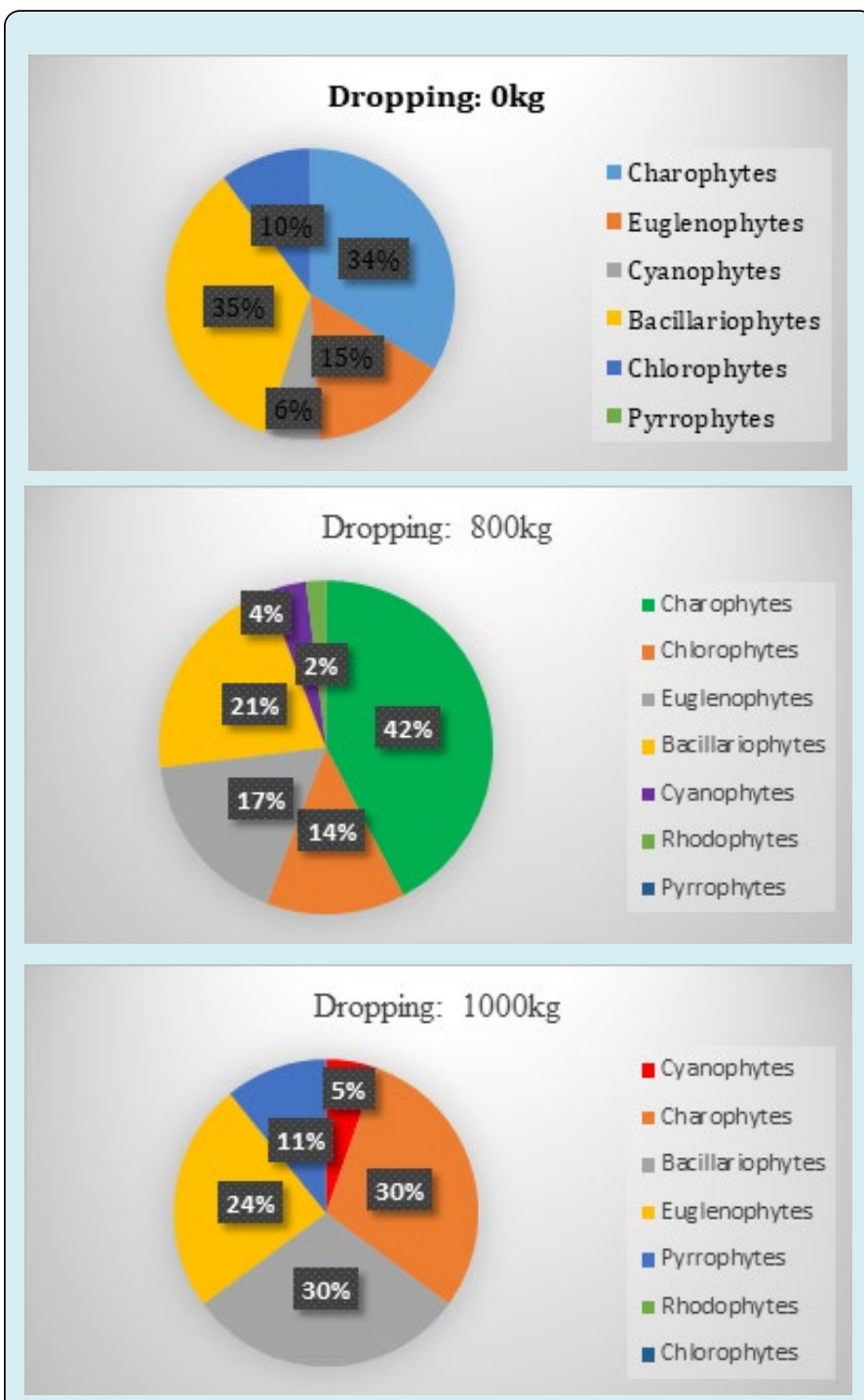

Figure 1: Specific distribution of phytoplankton as a function of dropping dose.

\section{Dynamics of Phytoplankton Density as a Function of Dropping Dose}

The evolution of phytoplankton density as a function of manure dose is illustrated in Figure 2, which shows that the trend, profile and rate of change in density were generally comparable between treatments.

Regardless of the test period, the lowest phytoplankton density values were observed in unfertilized ponds and the highest in ponds fertilized with the highest dose of chicken dropping (1000 kg/ha).

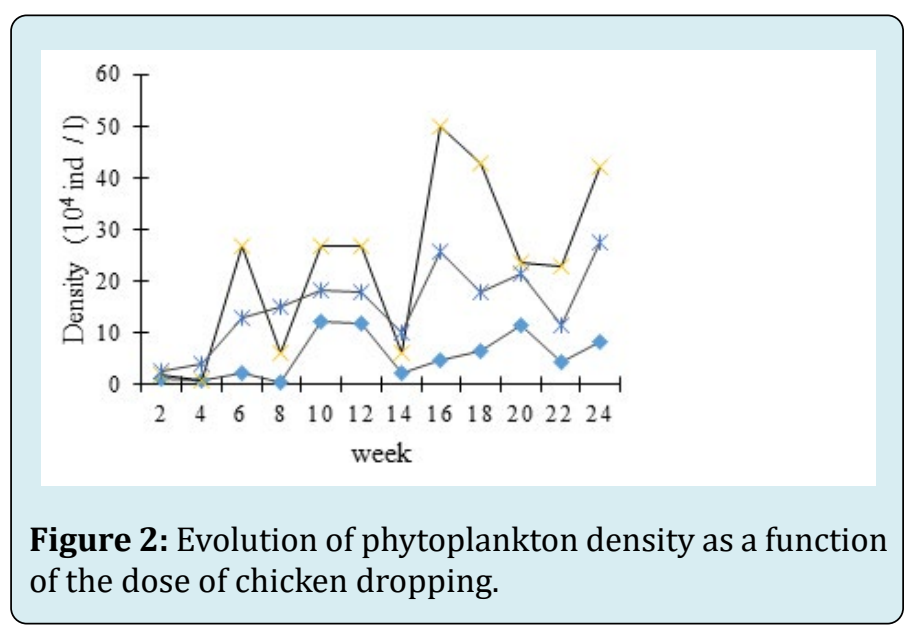

Statistical analysis conducted on phytoplankton density showed that there was no significant difference $(p<0.05)$ between ponds fertilized at the 800 and $1000 \mathrm{~kg}$ of chicken droppings (Figure 3).

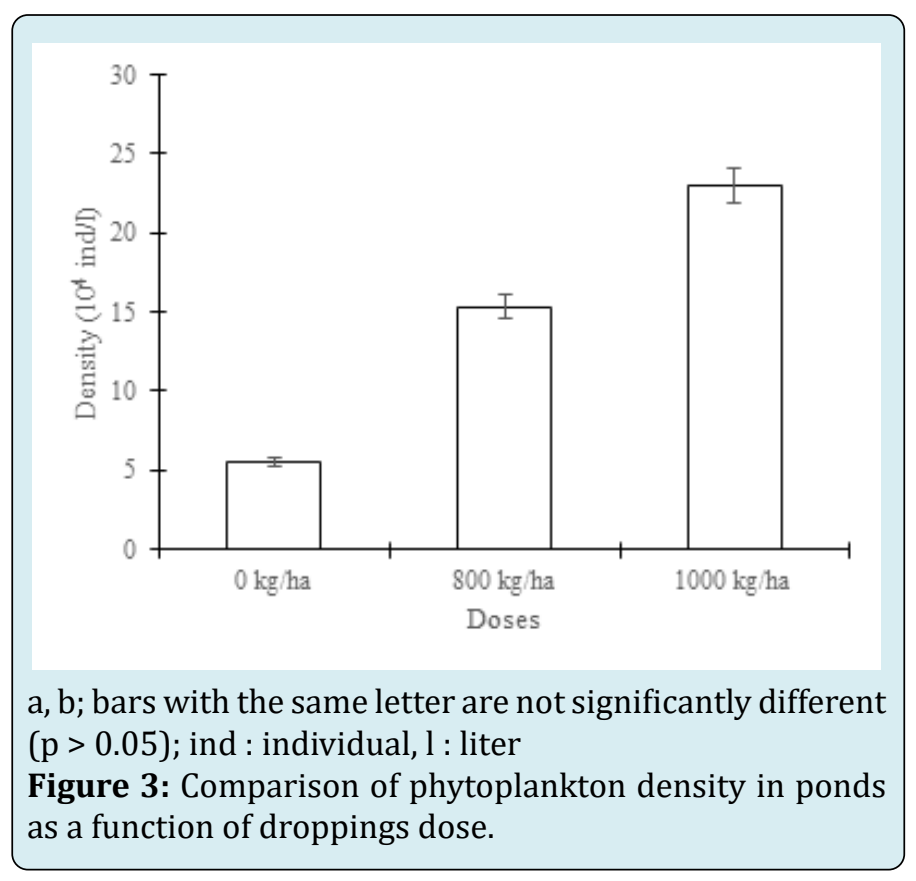

\section{Phytoplankton Diversity Index}

The index Shannon \& Weaver diversity and equitability summarized in Table 2 showed a decrease with increasing 
fertilizer dose. Thus, the highest values of Shannon's index and equitability were recorded in unfertilized ponds and the lowest in ponds fertilized with the $1000 \mathrm{~kg}$ of chicken droppings.

\begin{tabular}{|c|c|c|c|}
\hline \multirow{2}{*}{ Diversity index (bits / ind) } & \multicolumn{3}{|c|}{ Chicken droppings } \\
\cline { 2 - 4 } & $\mathbf{0}(\mathbf{k g} / \mathbf{h a})$ & $\mathbf{8 0 0}(\mathbf{k g} / \mathbf{h a})$ & $\mathbf{1 0 0 0}(\mathbf{k g} / \mathbf{h a})$ \\
\hline Shannon \& Weaver & 2,70 & 2,17 & 2,10 \\
\hline Equitabilité J & 0,52 & 0,45 & 0,44 \\
\hline
\end{tabular}

Table 2: Index of Phytoplankton Species Diversity.

Correlation between Physicochemical Characteristics of Water and Phytoplankton Density as a Function of Fertilizer Dose: Correlations between physicochemical characteristics of water and phytoplankton density (Table 3) showed that phytoplankton density is negatively and highly correlated $(\mathrm{p}<0.01)$ with electrical conductivity, $\mathrm{pH}$, dissolved oxygen, nitrite and nitrate in the control treatment. Conversely, phytoplankton density is positively and strongly correlated to transparency, dissolved oxygen and $\mathrm{pH}$ in the treatment at the dose of $800 \mathrm{~kg} / \mathrm{ha}$ chicken droppings.

\begin{tabular}{|c|c|c|c|}
\hline \multirow{2}{*}{ Physicochemical Characteristics } & \multicolumn{3}{|c|}{ Density of Phytoplankton } \\
\cline { 2 - 4 } & $\mathbf{0 ~ k g}$ & $\mathbf{8 0 0} \mathbf{~ k g}$ & $\mathbf{1 0 0 0} \mathbf{~ k g}$ \\
\hline Transparency & $+0,061$ & $+0,966^{* *}$ & $+0,134$ \\
\hline Temperature & $+0,760^{* *}$ & $-0,815^{* *}$ & $-0,348$ \\
\hline $\mathrm{O}_{2}$ & $-0,994^{* *}$ & $+0,209$ & $+0,981^{* *}$ \\
\hline $\mathrm{pH}$ & $-0,750^{* *}$ & $-0,926^{* *}$ & $-0,075$ \\
\hline $\mathrm{NO}_{2}{ }^{-}$ & $-0,927^{* *}$ & $-0,283$ & $+0,165$ \\
\hline $\mathrm{NO}_{3}{ }^{-}$ & $-0,818^{* *}$ & $-0,359$ & $+0,233$ \\
\hline $\mathrm{PO}_{4}{ }^{-}$ & $+0,482$ & $-0,499$ & $-0,999^{* *}$ \\
\hline
\end{tabular}

**: significant correlation $\mathrm{p}<0.01$ (bilateral);

Table 3: Correlation between physicochemical characteristics of the water and phytoplankton density.

\section{Discussion}

Results on the effect of the droppings dose on the composition of the phytoplankton showed that species richness was higher in the unfertilized ponds (control treatment). This observation is probably related to changes in the physicochemical characteristics of the water influenced by fertilizer doses. Patrick [20] has shown that the diversity of an environment can decrease when it is polluted. According to Moss [21], this observation is due to the fact that only species capable of adapting to a strong enrichment of the environment in nutrients and to an environment presenting extreme conditions according to the physico-chemistry, will have an advantage in eutrophic conditions. The population of species incapable of adapting decreases and that of species capable of adapting increases, and the proportions of taxa as a function of trophic level are thus modified according to the populations of these taxa. Radji, et al. [7] also reported that the dynamics of phytoplankton populations are influenced by the physicochemical characteristics of the water.

The highest species richness in the control treatment shows the high variability of the species living in an environment. These results are verified by Shannon \& Weaver's indices of diversity and lower equitability in fertilized ponds. The imbalance observed in the fertilized environments would come from a continuous supply of fertilizers that modify the physicochemical characteristics of the water. Moreover, only species that are cosmopolitan or have a broad ecological spectrum are capable of adapting to variations in the physicochemical characteristics of water.

The species richness of the genus Spirogyra was highest in the treatment at the dose of $800 \mathrm{~kg} / \mathrm{ha}$ droppings. This result is contrary to those reported by Nguetsop [22]; Folefack [23] who found spirogyra in abundance in flowing waters with high organic pollution. The difference between these results would be due to the fact that species belonging to this genus are found in various ecological conditions; in lakes, ponds and marshes, in oligo- to eutrophic waters with a $\mathrm{pH}$ ranging from 5 to 8 [24].

The number of phytoplankton phytoplankton branches found in fertilized ponds was higher compared to the four 
phytoplankton phytoplankton branches (Cyanophytes, Chlorophytes, Euglenophytes, Bacillariophytes) obtained by Ponce-Palafox, et al. [25] on the effect of organic and chemical fertilization on the production of phytoplankton and carp in polyculture. The same is true with results obtained by Agadjihouede, et al. [26] who recorded a single branch (Chlorophytes) in tanks fertilized with chicken droppings and cow dung). The observed differences are thought to be due to the direct effect of fertilizer doses on the physicochemical characteristics of the water. Indeed, the concentrations of nitrites $(7.92 \pm 0.05 \mathrm{mg} / \mathrm{l})$, nitrates $(8.03$ $\pm 0.2 \mathrm{mg} / \mathrm{l})$ and phosphates (4.68 $\pm 0.05 \mathrm{mg} / \mathrm{l})$, which are essential for the development of phytoplankton in our tests, were significantly higher.

Phylum of phytoplankton such as Cyanophytes, Chlorophytes, Euglenophytes and Bacillariophytes identified were similar to those observed by several authors in freshwater $[4,9,25,26]$. This observation can be justified by their cosmopolitan character and in particular by their relative abundance in African freshwater [16].

Rhodophytes and Pyrrophytes with very low diversity were only present in the fertilized ponds. This would be due to the fact that these branches are mostly marine species and their presence in fresh water is limited to about thirty infrequent genus. These taxa are therefore very rare in Sudanian waters [16].

Species in the Charophytes phylum as well as Bacillariophytes were more abundant in the control treatment. The high proportion of species belonging to these branches would be linked to the poor tolerance of these species to pollution and/or the fact that they have been little consumed by zooplankton despite their high nutritional quality [27] due to the significantly low zooplankton density in this treatment.

Rhodophytes were very poorly diversified in terms of species in the fertilized ponds, thus justifying the mesotrophic nature of the fertilized waters. Indeed, Rhodophytes proliferate better in oligotrophic aquatic environments where the water is little polluted [28].

Phytoplankton density showed that the number of cells per unit volume obtained was significantly higher in fertilized ponds. This observation would be due to the nutrient richness of the environment in relation to the dose of droppings. Moreover, correlations showed that the phytoplankton density was highly and significantly $(\mathrm{p}<0.01)$ negative to electrical conductivity, $\mathrm{pH}$, dissolved oxygen, nitrite and nitrate in the control treatment.

\section{Conclusion}

The development of phytoplankton in ponds has shown that species richness, species distribution, density and dynamics have been affected by the dose of chicken droppings. The species richness has inversely evolved with respect to the droppings dose. On the other hand, phytoplankton density increased with fertilizer application. It was noted that the proportion of Cyanophytes, phylum not very useful for fish, decreased with the chicken intake in favour of Bacillariophytes having an important nutritional quality for fish. For optimal production of phytoplanktonophagous fish, the dose of $1000 \mathrm{~kg}$ could be used.

\section{References}

1. Hasnaoui M, Souissi S, Balvay G (2007) Distribution spatio-temporelle du phytoplancton dans un etang d'alevinage (station de la Deroua, Beni-Mellal, Maroc). Sud sciences \& technologies, pp: 12.

2. Barbe J, Schlumberge O, Bouretz N (1999) Utilisation du phytoplancton pour estimer la production piscicole potentielle des etangs. Bull Fr Peche Piscic 355: 387-402.

3. Kemka N, Njine T, Zebaze Togouet SH, Niyitegeka D, Nola M, et al. (2004) Phytoplancton du lac municipal de Yaounde (Cameroun) : Succession ecologique et structure des peuplements. Revue des sciences de l'eau / Journal of Water Science 17(3): 301-316.

4. Njine T, Kemka N, Zebaze Togouet SH, Nola M, Niyitegeka D, et al. (2007) Peuplement phytoplanctonique et qualite des eaux en milieu lacustre anthropise: cas du lac municipal de Yaounde (Cameroun). African Journal of Science and Technology Science and Engineering Series 8(1): $39-51$.

5. Nguetsop VF, Fonkou T, Dekoum VM, Ndemafo MN, Pinta JY (2007) Relationships between algae taxa and physicochemical characteristics of water in wetlands and water bodies. Cameroon J Exper Biol 3(2): 70-79.

6. Fonge BA, Tening AS, Egbe EA, Yinda GS, Fongod AN, et al. (2012) Phytoplankton diversity and abundance in Ndop wetland plain, Cameroon. African Journal of Environmental Science and Technology 6(6): 247-257.

7. Radji R, Bandje A, Issifou L, Edorh $\mathrm{T}$, Kouami $\mathrm{K}$ (2013) Diversite et dynamique des assemblages phytoplanctoniques dans les ecosystemes aquatiques au Sud du Togo. Afrique Science 9(2): 67-77.

8. Greenberg A (1985) Standard methods for the examination of water and waste water, $16^{\text {th }}($ Edn.), American Public Health Association, Washington, pp: 1268. 
9. Nguetsop VF, Fonkou T, Lekeufack M, Pinta JY (2009) Assemblages dalgues et relations Avec quelques parametres environnementaux dans deux sites marecageux de lOuest-Cameroun. Revue des Sciences de l'Eau 22 (1) 15-27.

10. Bourrelly P, Manguin E (1952) Algues d'eau douce de la Guadeloupe et dépendances, SEDES, Paris, pp : 276.

11. Compere P (1974) Algues de la region du lac Tchad. IICyanophycees. Cah ORSTOM ser Hydrobiol 8(3/4): 165198.

12. Coute A, Rousseli G (1975) Contribution a Ietude des algues d'eau douce du moyen Niger (Mali). Bull Mus Nat Hist Nat Pari Ser Bot 21: 73-176.

13. Compere P (1976) Algues de la region du lac Tchad. V. Chorophycophytes 1. Cah ORSTOM ser Hydrobiol 10(2): 77-118.

14. Compere P (1977) Algues de la rdu lac Tchad. VIIChorophycophytes 3e partie : Desrnidiees Cah ORSTOM Hydrbiol 11(2): 77-177.

15. Gasse F (1980) Les diatomEes lacustres plio-pleistocene $\mathrm{du}$ gadeb (Ethiopie). Systematique, paleoecologie, biostratigraphie. Rev Algol 1 : 62-249.

16. Iltis A (1980) Les algues. La Flore et Faune Aquatiques de l'Afrique Sahelo-Soudanienne (Tome I). In: Durand JR, LEvEque C, (Eds.), Collection Initiations Doc Tech, ORTOM, pp: 44: 9-61.

17. Gasse F (1983) Diatom assemblages in East Africa: classification, distribution and ecology. Rev Hydrobiol Trop 16: 3-34.

18. Krammer k, Lange-Bertalot H (1991) Süsswasserflora von Mitteleuropa. Bacillariophyceae. Teil 3: Centrales, Fragilariaceae, Eunotiaceae. Fischer verlag, Stuttgart, Allemagne, pp: 598.

19. Lund JWG, Kilpling C, Le Cren ED (1958) The inverted microscope method of estimating algal numbers and the statistical basis of estimation by counting. Hydrobiology 11: $143-170$.

20. Patrick R (1973) Use of algae, especially diatoms, in the assessment of water quality. In: Cairns J, Dickson KL, (Eds.), Biological Methods for the Assessment of Water Quality, ASTM STP 528, American Society for Testing and Materials, Philadelphia, pp: 76-95.

21. Moss B (1998) Ecology of fresh waters: man and medium, past to future. Blackwell Science, Oxford, pp : 557.

22. Nguetsop VF (1990) Flore algale de quelques marecages à Cyrtosperma senegalense dans la région de Yaoundé. Mémoire de Maîtrise, Univ Yaoundé, pp: 88.

23. Folefackc A (1989) Étude de la flore algale du lac municipal de Yaoundé. Mémoire de Maîtrise, Univ Yaoundé, pp: 77.

24. Forster K (1982) Das Phytoplankton des Siisswassers. Conjugatophyceae: Zygnematales and Desmidiales (Zygnemataceae). DieBinnengewâsser, E. Schweizerbart, Stuttgart 8(1) : 16 .

25. Ponce-Palafox JT, Arredondo-Figueroa JL, MedinaCarrillo F, Castillo-Vargasmachuca S, Regalado-De Dios M (2010)The effect of chemical and organic fertilization on phytoplankton and fish production in carp (cyprinidae) polyculture system. Rev Bioscienc 1(1): 1171-1186.

26. Agadjihouede H, Bonou CA, Chikou A, Laleye P (2010) Production comparee de zooplancton en bassins fertilises avec la fiente de volaille et la bouse de vache. International Journal of Biological and Chemical Sciences 4(2): 432-442.

27. Lam CWY, Silvester WB (1979) Growth interaction among blue-green algae. Hydrobiology 63(2): 135-143.

28. Adjahouinou DC, Liady ND, Fiogbe DE (2012) Diversite phytoplanctonique et niveau de pollution des eaux du collecteur de Dantokpa (Cotonou-Benin) International Journal of Biological 6(5): 1938-1949. 